\title{
Eficácia do método Pilates e do biofeedback manométrico em mulheres na menopausa com incontinência urinária
}

\section{Efficacy of the Pilates method and the manometric biofeedback in menopausal women with urinary incontinence}

\author{
Elisa Pinheiro Schrader ${ }^{1}$, Juliana Cristina Frare ${ }^{2}$, Karen Andréa Comparin ${ }^{3}$, Cristina Diamante ${ }^{4}$, \\ Beatriz Gavassa de Araújo ${ }^{5}$, Caroline Danielli ${ }^{5}$ Letícia Dubay Murbach $^{5}$
}

\begin{abstract}
Resumo
A Incontinência Urinária é toda perda involuntária de urina, classificada em Incontinência Urinária de Esforço, de Urgência e Mista. A prevalência desta disfunção afeta negativamente a qualidade de vida das pessoas, sendo o tratamento fisioterapêutico recomendado como primeira opção. Dentre as técnicas fisioterapêuticas, encontram-se o biofeedback e o Método Pilates. O objetivo do estudo foi verificar a eficácia do tratamento fisioterapêutico para incontinência urinária com o método Pilates e o biofeedback manométrico em mulheres na menopausa. O estudo foi realizado na clínica da Universidade Estadual do Oeste do Paraná e a amostra foi constituída por 14 mulheres, divididas em dois grupos. O grupo 1 foi submetido ao tratamento com o biofeedback e o grupo 2 com o Método Pilates. Ambos realizaram trinta e duas sessões e foram avaliados antes e após os tratamentos considerados curto (após vinte e duas sessões), médio (após trinta e duas sessões) e longo prazo (após dois meses sem intervenções), por meio do Questionário de Incontinência Urinária ICIQ_SF e de uma Ficha de Coleta de Dados. Ambos os grupos apresentaram resultados estatisticamente significativos quando realizada a comparação intra-grupos em relação à frequência das perdas urinárias mensais, força das fibras rápidas do assoalho pélvico, gravidade da incontinência urinária e o impacto desta sobre a qualidade de vida das mulheres. $\mathrm{Na}$ duração das contrações mantidas, apenas o biofeedback apresentou diferenças estatisticamente significativas. Não houve diferença estatisticamente significativa entre os grupos. Ambas as técnicas propostas foram eficazes nas variáveis analisadas, exceto na duração das contrações mantidas.

Palavras chave: Incontinência urinária. Modalidades de fisioterapia. Qualidade de vida.
\end{abstract}

\begin{abstract}
The urinary incontinence is an involuntary loss of urine, classified as stress, urge and mixed urinary incontinence. The prevalence of this dysfunction negatively affects people's life quality and the physiotherapeutic treatment is recommended as the first option. The biofeedback and the Pilates method are among the physiotherapeutic techniques. The aim of this study was to verify the efficacy of the physiotherapeutic treatment for urinary incontinence using the Pilates Method and the manometric biofeedback in menopausal women. The study was carried out in the clinic of the State University of Western Paraná and the sample was made up with 14 women, divided in two groups. Group 1 was submitted to the treatment with biofeedback, and group 2, with the Pilates method. Both performed thirty-two sessions and were evaluated before and after the short (after twenty-two sessions), medium (after thirty-two sessions) and long-term treatments (after two months without interventions), by using the Urinary Incontinence Questionnaire ICIQ_SF and a Data Collection Form. Both the groups presented statistically significant results in the intra-group comparison, related to the frequency of the monthly
\end{abstract}

\footnotetext{
${ }^{1}$ Fisioterapeuta, residente de Fisioterapia em Uroginecologia e Obstetrícia Funcional pela Universidade Estadual de Londrina; Londrina, Paraná, Brasil. E-mail: elisaschrader@hotmail.com

${ }^{2}$ Doutorado em Estomatopatologia pela Universidade Estadual de Campinas. Docente do Colegiado do Curso de Fisioterapia na Universidade Estadual do Oeste do Paraná; Cascavel, Paraná, Brasil.

${ }^{3}$ Fisioterapeuta. Professora assistente do curso de Fisioterapia da Universidade Estadual do Oeste do Paraná; Cascavel, Paraná, Brasil.

${ }^{4}$ Doutorado em Advanced Sciences in Rehabilitation Medicine, Università degli Studi di Roma Tor Vergata; Roma, Itália. Docente do Colegiado do Curso de Fisioterapia na Universidade Estadual do Oeste do Paraná; Cascavel, Paraná, Brasil.

${ }^{5}$ Fisioterapeuta pela Universidade Estadual do Oeste do Paraná; Cascavel, Paraná, Brasil.
} 
urinary losses, the strength of the pelvic floor rapid fibers, the severity of the urinary incontinence and its impact on women's life quality. About the duration of the maintained contractions, only the biofeedback presented statistically significant differences. There was no statistically significant difference between the groups. Both the techniques were efficient in the variables analyzed, except in the duration of the maintained contractions.

Keyword: Urinary incontinence. Physical therapy modalities. Quality of life.

\section{Introdução}

De acordo com a International Continence Society (ICS) e com a International Urogynecological Association (IUGA), a incontinência urinária (IU) é reconhecida como uma disfunção, definida pela queixa de perda involuntária de urina, podendo ser classificada conforme os eventos apresentados pela paciente em: incontinência urinária de esforço (IUE), incontinência urinária de urgência (IUU) e incontinência urinária mista (IUM) (HAYLEN et al., 2012).

A IUE é o tipo mais comum, que acomete cerca de $50 \%$ das mulheres com IU. É definida como a queixa de perda involuntária de urina quando a pressão intra-abdominal e, consequentemente, a pressão intravesical, excedem a pressão de fechamento uretral máxima. Neste tipo de IU a perda de urina ocorre durante esforços, como no exercício, tosse, espirro, risada, saltos, caminhada, corrida e levantamento de peso. Sua etiologia caracteriza-se por ser multifatorial, sendo a causa mais comum a disfunção dos músculos do assoalho pélvico (MAP) (GUIRRO, 2009).

A IUU ocorre quando há o desejo repentino e forte de urinar sem a capacidade de controlar o mecanismo de micção, normalmente causado por contrações involuntárias do músculo detrusor (JUSTINA, 2013).

Por fim, a IUM é a associação dos dois tipos descritos anteriormente (JUSTINA, 2013).

A IU decorre de múltiplos fatores, como disfunção dos MAP, distúrbios da inervação, anormalidades nas estruturas conectivas que sustentam e estabilizam as vísceras dentro da cavidade abdominal e alterações posturais que afetam o equilíbrio pélvico, prejudicando a continência. Os fatores predisponentes à IU podem estar relacionados à paridade, à via de parto, à idade, ao peso do recém-nascido, à menopausa, à obesidade e à genética. Atividades físicas de alto impacto também podem estar relacionadas à perda involuntária de urina (AGOSTINHO; BERTOTTO, 2009; ALBA et al., 2011).

A prevalência da IU feminina no Brasil é elevada e merece atenção por parte dos profissionais da área da saúde (JUSTINA, 2013). Apesar de não colocar diretamente a vida das pessoas acometidas em risco, a IU pode afetar negativamente sua qualidade de vida (QV) em diversos aspectos, tanto sociais e psicológicos, como físicos, pessoais e sexuais (FITZ et al., 2012a).

Atualmente existe um crescente interesse para as abordagens não operatórias e não farmacológicas no tratamento da IU em decorrência dos bons resultados encontrados, do baixo custo e dos poucos efeitos adversos, sendo o tratamento fisioterapêutico recomendado como primeira opção, apresentando nível A de evidência científica (GIRALDO et al., 2009; QASEEM et al., 2014; RESENDE; STÜPP, 2015). Dentre as técnicas fisioterapêuticas utilizadas no tratamento da IU, destaca-se o uso do biofeedback (BF) manométrico e as técnicas posturais, como o método Pilates (MP).

O BF é um método de treinamento dos músculos do assoalho pélvico (TMAP) que utiliza um dispositivo vaginal para medir a pressão da contração muscular e a atividade elétrica no músculo, fornecendo informação sobre o grau de contração à mulher através de meios visuais ou auditivos (HERDERSCHEE et al., 2011). É considerado adequado para pacientes com IU que apresentam déficit da consciência dos MAP, incapacidade de contrair ou relaxar voluntariamente este grupamento muscular ou intensidade de contração muito baixa (AGOSTINHO; BERTOTTO, 2009), uma vez que o intuito do BF é tornar a paciente consciente da função muscular por meio da captação da atividade muscular, aumentar a motivação da paciente durante o treinamento e, pela repetição da tarefa correta, proporcionar o automatismo necessário para o sucesso terapêutico (RESENDE; STÜPP, 2015).

O MP, por sua vez, é um programa completo de condicionamento físico e mental, cujos benefícios envolvem o ganho de força muscular do diafragma pélvico, a flexibilidade muscular, com consequente melhora na QV (CORREA; MOREIRA; GARCEZ, 2015; DINIZ et al., 2014; MARÉS et al., 2012). A técnica, criada por Joseph Pilates, consiste em exercícios isotônicos e isométricos e tem por característica a exigência dos músculos abdominais, 
músculos estabilizadores da coluna vertebral, íliopsoas, quadríceps, e também da região lombossacral e do assoalho pélvico (AP). Tais músculos compõem o que Joseph denominou de "centro de força", promovendo a estabilização estática e dinâmica do corpo (CURI, 2009; SILVA; MANNRICH, 2009).

A prevalência da IU feminina é elevada e afeta negativamente a $\mathrm{QV}$ das pacientes acometidas. $\mathrm{O}$ tratamento não cirúrgico é considerado como primeira opção na maioria dos casos. Em virtude disso, é fundamental a presença de estudos científicos que comprovem a eficácia de diferentes métodos de tratamento fisioterapêutico para IU, como o MP e o $\mathrm{BF}$, a fim de aprimorar a abordagem conservadora e consequentemente garantir um melhor resultado para as pacientes.

Nesse sentido, o estudo tem como objetivo verificar a eficácia do tratamento fisioterapêutico para IU com o MP e o BF manométrico em mulheres na menopausa, bem como mensurar, registrar e comparar a força dos MAP, através do BF manométrico pressórico no pré e pós-tratamento fisioterapêutico e comparar a eficácia do tratamento fisioterapêutico com o MP e o BF manométrico sobre a QV de mulheres com IU.

\section{Material e Método}

Este estudo é descritivo de caráter quantitativo do tipo ensaio clínico e foi realizado na Clínica de Fisioterapia da Universidade Estadual do Oeste do Paraná - Unioeste, mediante autorização da direção da mesma, no período de abril de 2014 a setembro de 2015 . Este trabalho foi aprovado pelo Comitê de Ética em Pesquisa Envolvendo Seres Humanos da Universidade Estadual do Oeste do Paraná sob parecer número 898.545 e CAAE número 28746214.8.0000.0107.

A amostra foi constituída por 14 mulheres com queixa de perda urinária involuntária. Os critérios de inclusão selecionados foram: ser do sexo feminino, apresentar IU, relatar os sintomas há pelo menos um ano, não apresentar prolapsos de órgãos pélvicos graus três e quatro, classificados de acordo com o sistema POP-Q (Pelvic Organ Prolapse Quantification System) (BUMP et al., 1996), estar no período da menopausa, ter disponibilidade para participar do tratamento e assinar o Termo de Consentimento Livre e Esclarecido (TCLE). Foram critérios de exclusão: ter realizado cirurgia há menos de cinco anos e qualquer tratamento fisioterapêutico para incontinência, estar em período reprodutivo, apresentar acometimento neurológico, ser tabagista, apresentar quadro de infecção vaginal e não comparecer às sessões de tratamento por duas vezes consecutivas sem justificativa.

Inicialmente foi realizada uma busca aleatória por mulheres que referiam apresentar perda involuntária de urina. Estas responderam a um questionário inicial com dados pessoais para seleção da amostra de acordo com os critérios de inclusão. Foi realizada uma palestra explicativa pela acadêmica responsável pela pesquisa, com supervisão da orientadora, com o objetivo de repassar maiores informações sobre a patologia, metodologia e objetivos da pesquisa, e para esclarecimento de possíveis dúvidas. Na mesma data, as voluntárias assinaram o TCLE (APÊNDICE 01), responderam à Ficha de Coleta de Dados (APÊNDICE 02) e o Questionário de Incontinência Urinária ICIQ_ SF (ANEXO 01) que foram coletados individualmente, onde a acadêmica questionava e a paciente respondia de acordo com os seus sintomas. Nesta mesma etapa, a amostra foi dividida nos grupos de tratamento.

O ICIQ_SF, validado para o português, é um questionário utilizado para avaliar IU, composto por seis questões que avaliam a frequência, a gravidade da perda urinária e o impacto da IU na vida diária, além de uma sequência de oito itens autodiagnósticos, relacionados às causas ou situações de IU, que não são pontuados. A soma dos escores das questões três, quatro e cinco varia de zero a 21 , sendo que quanto mais elevado o escore total, maior é a gravidade da IU. Já o impacto na vida diária é definido de acordo com o escore da questão cinco: (0) nada, (1-3) leve, (4-6) moderado, (7-9) grave, e (10) muito grave (TAMANINI et al., 2004).

As voluntárias foram divididas aleatoriamente por meio de um sorteio em dois grupos de tratamento: Grupo 1 (G1), composto por sete mulheres tratadas com BF, e o Grupo 2 (G2), composto também por sete mulheres tratadas pelo MP. Foram então realizadas trinta e duas sessões de atendimento divididas em duas etapas. A primeira realizada com uma frequência de duas vezes por semana durante onze semanas, totalizando vinte e dois atendimentos, e a segunda etapa com frequência de uma vez por semana durante dez semanas, totalizando dez atendimentos.

$\mathrm{Na}$ primeira sessão, realizou-se individualmente uma avaliação clínica de cada paciente por meio de exame físico e mensuração da atividade contrátil dos MAP. Esta etapa teve como objetivo verificar a pressão gerada pela contração muscular, bem como se todas as pacientes possuíam percepção dos MAP para correta execução dos exercícios propostos. 
Para avaliação, utilizou-se um perineômetro de pressão da marca Quark, modelo Perina ${ }^{\circledR}$ 996-2, que registrava os potenciais de ação das contrações dos MAP e traduzia sua intensidade em sinais visuais, por meio de escala numérica graduada de 0 a 46,4 $\mathrm{mmHg}$.

A voluntária foi posicionada em uma maca em decúbito dorsal (DD) com membros inferiores (MMII) flexionados e abduzidos e foi solicitada uma contração voluntária dos MAP. Na sequência, foi introduzida no canal vaginal a sonda pressórica revestida por preservativo de látex e lubrificada por gel a base de água. Novamente foram solicitadas três contrações rápidas intervaladas dos MAP e anotados os valores alcançados. Em seguida, foram pedidas três contrações mantidas e intervaladas, sendo registrados os tempos máximos que a paciente sustentava as contrações. Como medidas de proteção e higiene, foram utilizadas luvas descartáveis durante todo o procedimento e o local e os instrumentos foram higienizados com álcool $70 \%$ antes e após cada avaliação.

Os atendimentos do G1 foram realizados individualmente. As pacientes eram submetidas ao tratamento por meio dos exercícios de contração dos MAP. O equipamento utilizado era um perineômetro de pressão. O monitoramento das contrações foi realizado por uma sonda vaginal com sensor que detecta as contrações dos MAP. Durante todo o procedimento, a paciente era orientada a observar a escala numérica/ luminosa do equipamento simultaneamente à execução dos exercícios.

A sessão de tratamento foi dividida em três fases: dez minutos de contrações dos MAP, cinco minutos de descanso para relaxamento da musculatura e dez minutos de exercícios novamente, totalizando vinte e cinco minutos de atendimento. Na primeira fase de dez minutos, a paciente realizava os exercícios em DD com os MMII apoiados na maca, fletidos e abduzidos. $\mathrm{Na}$ segunda fase, a posição era em decúbito lateral (DL) com os MMII fletidos e aduzidos. A sequência de exercícios foi realizada, inicialmente, com dez contrações rápidas e intervaladas, com a finalidade de exercitar as fibras fásicas. Posteriormente, com dez contrações sustentadas e intervaladas, com o objetivo de trabalhar as fibras tônicas. Ao final, a paciente posicionava-se novamente em DD com os MMII em flexão e abdução na maca para retirada da sonda vaginal. O local e instrumentos eram higienizados com álcool $70 \%$ ao se encerrar cada sessão.

O G2 foi submetido a um protocolo de atendimento no qual foram utilizados exercícios do MP solo, para fortalecer o centro de força (principalmente abdômen e AP). As pacientes eram orientadas sobre a forma correta de execução dos exercícios e supervisionadas durante todo o treinamento. Durante a sessão, oito exercícios da lista abaixo eram executados em dez repetições cada, sempre associados à contração dos MAP. No início e ao final de cada sessão era realizada aferição da pressão arterial periférica como medida de segurança, impedindo a inicialização dos exercícios caso esta estivesse superior a 160/105 mmHg, conforme as Diretrizes Brasileiras de Hipertensão IV (SOCIEDADE BRASILEIRA DE HIPERTENSÃO, 2010). Sendo assim, a paciente era liberada da terapia e agendada para o atendimento em outro dia da semana. 1)Agachamento: Posição inicial do corpo em posição ortostática, pés afastados na largura dos quadris, membros superiores (MMSS) apoiados na cintura, posicionamento lombopélvico neutro. O movimento é realizado durante a expiração, com contração do centro de força, realizando uma flexão de joelhos, mantendo posicionamento neutro da coluna lombopélvica, e joelhos em rotação externa. Postura mantida durante uma inspiração com retorno ao posicionamento inicial durante a expiração.

2)Rolamento para baixo: Posição inicial do corpo em posição ortostática, MMII afastados na largura dos quadris, MMSS ao longo do corpo. Movimento de flexão segmentar anterior da coluna cervical, torácica e lombar, durante a expiração e contração do centro de força, mantendo os joelhos em extensão e MMSS pendentes ao corpo até o alcance das mãos no chão ou até o limite da paciente sem fazer compensações. Postura mantida durante uma inspiração e retorno à posição inicial na expiração.

3)Alongamento do gato: Posição inicial em quatro apoios, com as mãos embaixo dos ombros, os joelhos embaixo dos quadris e os MMII afastados na distância dos quadris. Pelve e coluna em posição neutra. A cabeça segue o alinhamento da coluna torácica. Movimento de articular a coluna, sem desalinhar mãos e pernas. Durante a expiração, realizar inclinação posterior da pelve e arredondamento da coluna, articulando de forma sequenciada do cóccix até a cabeça. Em seguida, inspirar mantendo a posição com os abdominais contraídos e sustentando a cabeça com os ombros relaxados. Na expiração, articular novamente a coluna de maneira sequenciada até a posição inicial.

4) Inclinação pélvica: Posição inicial em quatro apoios, com as mãos alinhadas com os ombros e os joelhos 
com os quadris, MMII afastados na distância dos quadris. Pelve e coluna na posição neutra. A cabeça segue o alinhamento da coluna torácica. Movimento de inclinação pélvica em que durante a expiração, deve-se inclinar posteriormente a pelve sem alterar a posição da coluna, em seguida inspirar mantendo a posição com os abdominais contraídos. $\mathrm{Na}$ expiração, retorno à posição inicial.

5)Abdução da perna de cima: Posição inicial em DL, com o membro inferior (MI) de baixo levemente fletido, membro superior (MS) de baixo embaixo da cabeça e o de cima apoiado na superfície para dar estabilidade, quadril alinhado com joelho e tornozelo. Movimento de abdução do MI de cima, mantendo-o em extensão e em alinhamento, com abdominais contraídos. Postura mantida durante uma inspiração e retorno à posição inicial na expiração.

6)Ponte: Posição inicial em decúbito dorsal (DD), MMII fletidos e separados na largura do quadril, MMSS ao longo do corpo. Movimento realizado durante a expiração com contração do centro de força, firmando a base e levantando o quadril da superfície. Postura mantida durante uma inspiração e retorno à posição inicial na expiração.

7)Centenas - pés na bola: Posição inicial em DD, quadril e joelhos a $90^{\circ}$, calcanhares apoiados em uma bola suíça, MMSS ao longo do corpo. Movimento de elevação de cabeça e região cervicodorsal em direção a bola durante a expiração, mantendo os MMSS em extensão ao longo do corpo, porém sem apoiá-los na superfície. Postura mantida durante uma inspiração e retorno à posição inicial na expiração.

8)Batidas com os dedos: Posição inicial em DD, com quadril e joelhos a $90^{\circ}$, MMSS ao longo do corpo. Movimento de encostar a ponta dos dedos do pé na superfície e voltar aos $90^{\circ}$, repetir alternadamente com MI direito e esquerdo. O movimento é realizado durante a expiração associado à contração do centro de força.

9) Levantamento do quadril com pés na bola: Posição inicial em DD, com os MMSS ao longo do corpo, quadril e joelhos a $90^{\circ}$, pés apoiados na bola suíça. Movimento de elevar o quadril durante a expiração associado à contração do centro de força e retornar à posição inicial.

10)Abdominal com extensão de MMII sobre a bola: Posição inicial em DD, com as mãos cruzadas atrás da cabeça, MMII fletidos a $90^{\circ}$ e calcanhares apoiados na bola suíça. Movimento de elevação da cabeça até as escápulas saírem do chão com MMII fletidos. Retorno realizado repousando a cabeça e estendendo os MMII.

11)Abdominal oblíquo: Posição inicial em DD, MMII fletidos com bola entre os joelhos, e mãos atrás da cabeça. Movimento de elevação da cabeça e escápula lateralmente, alternando entre os lados direito e esquerdo. Execução do exercício durante a expiração associado à contração do centro de força. 12)Alongamento de piriforme e quadril: Posição inicial em DD com um MI estendido sobre a bola suíça e o outro MI fletido e apoiado sobre o mesmo. Movimento de flexionar o MI estendido tracionando o quadril junto ao peito. Realizado durante a expiração associado à contração do centro de força. 13)Concha: Posição inicial do corpo ajoelhada, com MMSS estendidos à frente do corpo segurando a bola suíça. Durante as expirações, realiza-se o movimento de rodar o tronco para a direita e para a esquerda.

Com o término da primeira etapa do tratamento, as pacientes eram reavaliadas com o perineômetro pressórico utilizado na avaliação inicial e respondiam novamente ao Questionário de Incontinência Urinária ICIQ_SF. Este momento foi considerado como uma reavaliação em curto prazo (Pós 1). Iniciava-se então a segunda etapa do tratamento, em que eram realizadas as dez sessões finais seguindo o mesmo protocolo de tratamento de acordo com cada grupo. Concluídas as trinta e duas sessões de tratamento, as pacientes eram novamente reavaliadas, considerando-se, portanto, um momento de reavaliação em médio prazo (Pós 2). Recebiam, então, orientações verbais e escritas, por meio de um folder ilustrativo (APÊNDICE 03), para a continuidade da realização dos EMAP em domicílio.

Após dois meses de Exercícios dos Músculos do Assoalho Pélvico (EMAP) domiciliares, as pacientes de ambos os grupos foram convocadas para reavaliação por meio do perineômetro pressórico e do ICIQ_SF para a obtenção dos resultados finais, considerando-se o momento de reavaliação em longo prazo (Pós 3).

Coletados os dados, os mesmos foram agrupados e lançados em planilhas do programa Excel, sendo posteriormente analisados por meio de análise estatística descritiva (Média e Desvio Padrão), e avaliado o padrão de distribuição dos dados por meio do teste de Shapiro-Wilk e homogeneidade das variâncias por meio do teste de Levene. 
Uma vez que os dados foram coletados aleatoriamente, e que os pressupostos de normalidade e homogeneidade das variâncias foram conferidos, as variáveis 'Frequência das perdas miccionais mensais', 'Força da contração das fibras rápidas', 'Duração da manutenção da contração das fibras lentas', 'Gravidade da IU' e 'Impacto desta sobre a QV' foram analisadas por meio da Análise da Variância para Medidas Repetidas. As análises estatísticas foram realizadas no programa Statistica 7.0, utilizando um nível de significância de 0,05 .

\section{Resultados e Discussão}

A amostra inicial era composta por 27 mulheres, com $16(59,25 \%)$ participantes no grupo G1 e $11(40,74 \%)$ no grupo G2. Não concluíram o tratamento um total de 13 (48,14\%) participantes; destas, 9 (69,23\%) eram do grupo G1 e 4 (30,76\%) eram do grupo G2. Os motivos das desistências do grupo G1 foram: 2 (22,22\%) por reação alérgica ao látex do preservativo; 1 (11,11\%) por quadro de infecção urinária durante o tratamento; $1(11,11 \%)$ por procedimento cirúrgico urgente e não relacionado ao quadro e $5(55,55 \%)$ abandonaram o tratamento por motivos pessoais. Em relação ao grupo G2, 2 (50\%) mulheres interromperam sua participação no estudo por problemas ortopédicos e $2(50 \%)$ por justificativas pessoais.

De acordo com a Associação Portuguesa de Urologia (2013), a proporção de casos de IU é de três mulheres para cada homem entre os 45 e 65 anos. Na mulher, fatores como a história obstétrica e ginecológica tendem a ser considerados predisponentes dos distúrbios urinários. Além disso, com o avanço da idade ocorrem mudanças na composição corporal, como a diminuição das fibras colágenas, substituição de tecido muscular por tecido adiposo com consequente diminuição da força de contração dos MAP, diminuição da capacidade de armazenamento da bexiga e baixos níveis de estrógenos na pós-menopausa (JUSTINA, 2013; MORENO-VECINO et al., 2015).

Apesar de ser considerada como uma das principais afecções geriátricas (DOGAN et al., 2015), a média realizada de quatro estudos recentes, observados por meio de uma revisão bibliográfica, demonstra que $54,54 \%( \pm 7,87)$ das mulheres com IU apresentam idade entre 40 e 60 anos (JUSTINA, 2013). No presente estudo, todas as participantes estavam no período da menopausa e apresentavam média de idade de $55( \pm 9,32)$ anos.
Quanto ao tipo de IU, 12 (85,71\%) participantes apresentavam IUM e $2(14,29 \%)$ IUE, sendo que o G1 foi constituído por $5(71,42 \%)$ pacientes com sintomas de IUM e $2(28,58 \%)$ de IUE e o G2 por 7 (100\%) pacientes com IUM. No estudo de Amaral et al. (2015), a prevalência dos sintomas demonstrados pelas participantes que apresentavam idade média de $53,34( \pm 11,58)$ anos foi de IUU $(69,5 \%)$, seguida de IUE $(21,2 \%)$ e IUM $(9,3 \%)$ em uma amostra de 505 mulheres, apresentando divergência em relação ao presente estudo. Entretanto, Amaral et al. (2015) ainda observaram que as queixas relacionadas à IUU e IUM apresentaram-se maiores em mulheres com idade entre 50-59 anos, denotando similaridade com o presente estudo.

Em relação às variáveis analisadas, foram observadas a média da frequência das perdas miccionais mensais, a força de contração das fibras rápidas dos MAP, a duração da manutenção da contração das fibras lentas, a gravidade da IU e o impacto desta sobre a

QV das participantes, no pré e pós-tratamento em diferentes tempos (Pós 1, 2 e 3).

Quanto à frequência das perdas miccionais mensais de cada participante, não foi observada diferença estatisticamente significativa entre os grupos $\left(\mathrm{F}_{3,36}=0,3316 ; \mathrm{p}=0,803\right)$. Quando comparados os resultados das quatro avaliações intra-grupo (G1 e G2 separadamente), observou-se que, em ambos, a média de frequência das perdas miccionais da primeira avaliação (Pré) em relação à segunda (Pós1) apresentou redução estatisticamente significativa $(p=0,006$; $\mathrm{p}=0,003$, respectivamente). Após a terceira (Pós2) e quarta (Pós3) avaliações, foi observada estabilização do quadro, sem significância estatística entre estas últimas reavaliações $(p>0,05)$, com tendência a manutenção da redução dos valores, como observado na Tabela 1.

Foram também analisados os valores de contração rápida dos MAP, não sendo observada diferença estatisticamente significativa entre os grupos $\left(\mathrm{F}_{3,36}=0,084 ; \mathrm{p}=0,968\right)$. Ao comparar os resultados das quatro avaliações intra-grupo (G1 e G2 separadamente), observou-se que, em ambos, a média de contrações rápidas da primeira avaliação em relação à segunda apresentou redução estatisticamente significativa $(\mathrm{p}=0,033 ; \mathrm{p}=0,013$, respectivamente). Após a terceira e quarta avaliações, foi novamente observada estabilização do quadro, com manutenção 
das médias de contração rápida, sem significância estatística entre estas últimas reavaliações $(\mathrm{p}>0,05)$ (Tab. 2).

Em relação à duração das contrações mantidas, também não foi observada diferença estatisticamente significativa entre os grupos $\left(\mathrm{F}_{3,36}=1,864 ; \mathrm{p}=0,153\right)$. Na comparação intra-grupo (G1 e G2 separadamente), observou-se que no G1 a média de contrações mantidas da primeira avaliação em relação à segunda apresentou aumento estatisticamente significativo $(p=0,003)$, com manutenção elevada da média na terceira avaliação $(\mathrm{p}=0,029)$, mas diminuição da média de contração na quarta avaliação $(\mathrm{p}=0,266)$. No $\mathrm{G} 2$, foi possível observar um aumento da média das contrações das fibras lentas entre os tempos de avaliação, porém sem diferença estatisticamente significativa entre nenhuma das avaliações ( $p>0,05)$ (Tab. 3).

Tabela 1 - Médias e Desvios Padrão das frequências das perdas miccionais mensais avaliadas no pré e pós tratamento dos grupos G1 e G2

\begin{tabular}{lcccccc}
\hline & Grupo & \multicolumn{4}{c}{ Frequência perda mensal } & \multirow{2}{*}{ P } \\
\cline { 3 - 5 } & Pré & Pós 1 & Pós 2 & Pós 3 & \\
\hline \multirow{2}{*}{$\begin{array}{c}\text { Frequência de } \\
\text { perdas mensais }\end{array}$} & G1 & $\begin{array}{c}27,71 \\
( \pm 16,38)\end{array}$ & $\begin{array}{c}4 \\
( \pm 3,26)\end{array}$ & $\begin{array}{c}3,14 \\
( \pm 3,62)\end{array}$ & $\begin{array}{c}1,85 \\
( \pm 1,57)\end{array}$ & \\
& G2 & $\begin{array}{c}51,71 \\
( \pm 50,09)\end{array}$ & $\begin{array}{c}26 \\
( \pm 34,85)\end{array}$ & $\begin{array}{c}21,71 \\
( \pm 20,15)\end{array}$ & $\begin{array}{c}15,14 \\
( \pm 13,89)\end{array}$ & \\
\hline
\end{tabular}

Fonte: O autor

Os resultados apresentados pelo G2 demonstram divergência em relação ao estudo de Tournier (2011), que observou diferença estatisticamente significativa sobre a força dos MAP em cinco participantes tratadas com o MP em apenas três sessões.

Diniz et al. (2014) realizaram oito sessões de TMAP por meio do MP em seis mulheres com média de idade de 49,66 $( \pm 9,64)$ anos, e observaram diferenças estatisticamente significativas em $84 \%$ das voluntárias em relação à força muscular das fibras rápidas e lentas. No entanto, os autores observaram uma tendência de maior ganho de força das fibras musculares de contração rápida quando comparada ao das fibras lentas.

No presente estudo, não foi mensurada a força dos MAP durante as contrações mantidas, e sim o tempo de duração da contração. Entretanto, foi possível observar que o G2 apresentou melhor eficácia no aumento de força das fibras rápidas do que na capacidade se
Tabela 2 - Médias e Desvios Padrão da força de contração das fibras rápidas dos MAP no pré e pós tratamento dos grupos G1 e G2

\begin{tabular}{ccccccc}
\hline & Grupo & \multicolumn{4}{c}{ Contrações rápidas (mmHg) } & \multirow{2}{*}{ P } \\
\cline { 3 - 5 } & Pré & Pós 1 & Pós 2 & Pós 3 & \\
\hline & G1 & $\begin{array}{c}3,5 \\
( \pm 2,98)\end{array}$ & $\begin{array}{c}9,31 \\
( \pm 5,62)\end{array}$ & $\begin{array}{c}9,44 \\
( \pm 5,22)\end{array}$ & $\begin{array}{c}9,52 \\
( \pm 4,87)\end{array}$ & \\
$\begin{array}{c}\text { Contrações } \\
\text { rápidas } \\
(\mathbf{m m H g})\end{array}$ & $\mathrm{G} 2$ & $\begin{array}{c}5,96 \\
( \pm 5,60)\end{array}$ & $\begin{array}{c}12,8 \\
( \pm 10,44)\end{array}$ & $\begin{array}{c}13,2 \\
( \pm 7,01)\end{array}$ & $\begin{array}{c}13,09 \\
( \pm 7,85)\end{array}$ & 0,968 \\
& & & & & \\
\hline
\end{tabular}

Fonte: $\mathrm{O}$ autor

sustentação (endurance) das fibras lentas, estando em concordância com os achados de Diniz et al. (2014).

No G1, os achados encontrados demonstraram melhora na função muscular tanto em relação às contrações rápidas quanto às mantidas, corroborando com o estudo de Fitz et al. (2012b), que também utilizaram o BF em mulheres com média de idade de $58,1( \pm, 3)$ anos e observaram melhora estatisticamente significativa da função dos MAP após 12 sessões de tratamento em relação à força de contração das fibras lentas e rápidas, demonstrando a eficácia do $\mathrm{BF}$ a curto prazo. Santos (2015), em seu estudo de caso,

Tabela 3 - Médias e Desvios Padrão da duração das contrações mantidas dos MAP no pré e pós tratamento dos grupos G1 e G2

\begin{tabular}{ccccccc}
\hline & Grupo & \multicolumn{4}{c}{ Contrações mantidas (segundos) } & \multirow{2}{*}{ P } \\
\cline { 3 - 5 } & & Pré & Pós 1 & Pós 2 & Pós 3 & \\
\hline & $\mathrm{G} 1$ & $\begin{array}{c}11,43 \\
( \pm 13,47)\end{array}$ & $\begin{array}{c}23,33 \\
( \pm 15,59)\end{array}$ & $\begin{array}{c}20,01 \\
( \pm 11,12)\end{array}$ & $\begin{array}{c}15,68 \\
( \pm 5,68)\end{array}$ & \\
$\begin{array}{c}\text { Contrações } \\
\text { mantidas }\end{array}$ & & 4,36 & 6,92 & 10,54 & 11,3 & 0,153 \\
(segundos) & $\mathrm{G} 2$ & $( \pm 1,98)$ & $( \pm 1,98)$ & $( \pm 4,89)$ & $( \pm 3,28)$ & \\
\hline
\end{tabular}

Fonte: $\mathrm{O}$ autor

também relatou evolução no grau de força muscular dos MAP após 24 sessões de tratamento associando a cinesioterapia e o BF.

Desse modo, o BF demonstrou eficácia tanto no ganho de força de contração rápida quanto no aumento da duração das contrações mantidas, denotando melhor evolução do grau de força muscular do AP, quando comparado ao MP, e concordando com o estudo de Bertoldi, Ghisleri e Piccinini (2014), que comprovaram em seu estudo de revisão a importância do BF como aliado no tratamento da IU quando associado as contrações rápidas e lentas dos MAP, 
por permitir mudanças significativas na consciência perineal, ganho de força muscular, redução da perda urinária e melhora na QV das mulheres.

Segundo Ferreira e Santos (2009), em um programa de treinamento muscular, o incremento da força nas primeiras seis a oito semanas é predominantemente neural (frequência de ativação e recrutamento das unidades motoras), iniciando a fase de hipertrofia muscular somente após esse período. Portanto, apesar de ser generalizado a todos os tipos de fibras musculares, o processo de hipertrofia apresenta maior potencial nas fibras rápidas do que nas fibras lentas, o que pode justificar os resultados encontrados no grupo G2, que demonstraram melhor evolução terapêutica nas contrações rápidas do que nas mantidas.

No entanto, treinos específicos de resistência podem transformar as fibras rapidamente fatigáveis em fibras mais resistentes à fadiga, pelo aumento do número de mitocôndrias e densidade capilar. Isto é possível com a seleção de um programa de treinamento adequado para as fibras musculares lentas, que consiste em baixas cargas com elevado número de repetições (FERREIRA; SANTOS, 2009).

Deste modo, o BF foi considerado mais efetivo no ganho de hipertrofia das fibras lentas por ser possível monitorar as participantes do grupo G1 quanto ao tempo e a graduação da força de contração, uma vez que as mesmas eram orientadas a realizar a contração do AP com menor força, porém mantendo a contração por maior tempo. Tal controle torna-se impossível quando realizados os exercícios por meio do MP, denotando menor especificidade do método, com consequente ineficácia sobre esta variável.

Apesar de ser o método mais utilizado para mensurar a contração máxima e endurance dos MAP, o BF pressórico sem os eletrodos de superfície pode mascarar a força muscular do AP, uma vez que aumentos da pressão abdominal podem registrar valores maiores nas medidas de pressão. A mesma alteração pode ocorrer com a ativação indesejada dos adutores do quadril, rotadores externos e glúteos. Nesses casos, a observação visual simultânea desses músculos é indispensável (RESENDE; STÜPP, 2015). No presente estudo, foram fornecidas informações quanto à importância do isolamento dos MAP, além da observação clínica minuciosa por parte da terapeuta durante as avaliações, a fim de evitar alterações nos resultados.

Pelos sintomas apresentados pelas pacientes com IU, é possível perceber que esta afecção gera diversas repercussões (físicas, psicológicas, sociais e econômicas) que influenciam negativamente a QV (DOGAN et al., 2015). Para Fitz et al. (2012b), a melhora da funcionalidade dos MAP está diretamente associada à diminuição do número de perdas urinárias e, consequentemente, à melhoria do bem-estar destas mulheres. Baseado nisto, a gravidade da IU e o impacto desta sobre a QV das participantes também foram analisadas neste estudo por meio do ICIQ_SF.

Para mensurar a gravidade da IU, foram analisadas as questões três, quatro e cinco do ICIQ_SF. Pela análise dos resultados, foi possível observar que não houve diferença estatisticamente significativa entre os grupos $\left(\mathrm{F}_{3,36}=0,919 ; \mathrm{p}=0,442\right)$. Quando comparados os resultados das quatro avaliações intra-grupo (G1 e G2 separadamente), observou-se que, em ambos, a média de gravidade da primeira avaliação em relação à segunda apresentou redução estatisticamente significativa ( $<<0,0001 ; p<0,0001$, respectivamente). Após a terceira e quarta avaliações, foi observada estabilização do quadro, com manutenção das médias de gravidade da IU, sem significância estatística entre estas últimas reavaliações $(p>0,05)$, porém com manutenção da redução dos valores de gravidade da IU. Vale ressaltar que o G2 apresentou redução também significativa entre a segunda e a quarta avaliações $(p=0,009)$, indicando maior redução do grau de gravidade (Tab. 4).

O impacto da IU sobre a QV também foi analisado por meio do ICIQ_SF. Não foi observada diferença estatisticamente significativa entre os grupos novamente $\left(\mathrm{F}_{3,36}=0,714 ; \mathrm{p}=0,550\right)$. Quando comparados os resultados das quatro avaliações intragrupo (G1 e G2 separadamente), observou-se que, em ambos, o impacto da IU sobre a QV no Pré em relação ao Pós 1 apresentou redução estatisticamente significativa $(\mathrm{p}<0,0001 ; \mathrm{p}<0,0001$, respectivamente). Após a terceira e quarta avaliações, foi observada estabilização do quadro, com manutenção das médias de impacto da IU, sem significância estatística entre estas últimas reavaliações no G1 ( $\mathrm{p}>0,05)$, porém com redução significativa entre a segunda (Pós 1) e a quarta avaliações (Pós 3) (p=0,008) no G2 (Tab. 5).

Considerando que o impacto na vida diária é definido de acordo com o escore da questão cinco do ICIQ_SF em (0) nada, (1-3) leve, (4-6) moderado, (79) grave, e (10) muito grave (TAMANINI et al., 2004), foi possível observar que o G1 apresentou redução do impacto da IU de inicialmente moderada para leve logo na segunda avaliação. O G2, por sua vez, apresentou 
redução de grave para moderado impacto na segunda avaliação, permanecendo com diminuição dos valores até a quarta avaliação, com redução também significativa entre a segunda e a quarta avaliações $(\mathrm{p}=0,008)$.

Tais resultados relacionados ao G1 apresentam-se em concordância aos de Bertoldi, Ghisleri e Piccinini (2014), Fitz et al. (2012b) e Hirakawa et al. (2013), os quais também concluíram em seus estudos a eficácia do BF no aumento da QV das participantes.

Tabela 4 - Médias e Desvios Padrão dos resultados do ICIQ_SF em relação à gravidade da IU no pré e pós tratamento dos grupos G1 e G2

\begin{tabular}{ccccccc}
\hline & & \multicolumn{4}{c}{ ICIQ_SF gravidade } & \multirow{2}{*}{ P } \\
\cline { 3 - 6 } & Grupo & Pré & Pós 1 & Pós 2 & Pós 3 & \\
\cline { 3 - 6 } $\begin{array}{c}\text { ICIQ_SF } \\
\text { gravidade }\end{array}$ & G1 & 11,85 & 5,14 & 3,42 & 3 & \\
& & $( \pm 4,25)$ & $( \pm 2,73)$ & $( \pm 2,69)$ & $( \pm 2,94)$ & \\
& G2 & 12,85 & 8,71 & 6,42 & 5,57 & 0,442 \\
& & $( \pm 3,18)$ & $( \pm 2,98)$ & $( \pm 3,04)$ & $( \pm 2,63)$ & \\
\hline
\end{tabular}

Fonte: $\mathrm{O}$ autor

Correa, Moreira e Garcez (2015) também apresentam resultados positivos em relação à $\mathrm{QV}$ com o MP, em seu artigo de revisão, onde os autores afirmam a comprovação da técnica sobre o ganho de força nos MAP, aumento da flexibilidade e melhora considerável da patologia, bem como melhora da QV da paciente, reduzindo o volume de urina perdido ao esforço.

Lately (2002 apud BO; HERBERT, 2013) relatam a eficácia do MP no tratamento da IU, pois apesar de não treinar os MAP especificamente, estes são ativados de maneira indireta, permitindo que sua contração mantenha o AP contraposto ao aumento da pressão intra-abdominal que ocorre durante o exercício, prevenindo a perda urinária e fortalecendo essa estrutura muscular. Tal observação pode ser confirmada pelos achados encontrados no presente estudo em relação à força de contração das fibras rápidas, com repercussões na diminuição das perdas miccionais mensais, na gravidade do quadro de IU e na QV das participantes.

No entanto, não é possível visualizar a contração dos MAP durante a realização dos exercícios pelo MP, podendo este fator ser considerado uma limitação do método, uma vez que, conforme Agostinho e Bertotto (2009), apenas a orientação verbal dos EMAP pode levar a manobras que promovam incontinência em até
Tabela 5 - Médias e Desvios Padrão dos resultados do ICIQ_SF em relação ao impacto da IU no pré e pós tratamento dos grupos G1 e G2

\begin{tabular}{|c|c|c|c|c|c|c|}
\hline & \multirow{2}{*}{ Grupo } & \multicolumn{4}{|c|}{ ICIQ_SF impacto } & \multirow{2}{*}{$\mathbf{P}$} \\
\hline & & Pré & Pós 1 & Pós 2 & Pós 3 & \\
\hline \multirow{2}{*}{$\begin{array}{c}\text { ICIQ_SF } \\
\text { impacto }\end{array}$} & G1 & $\begin{array}{c}5,71 \\
( \pm 2,98)\end{array}$ & $\begin{array}{c}2,28 \\
( \pm 1,7)\end{array}$ & $\begin{array}{c}1 \\
( \pm 1,15)\end{array}$ & $\begin{array}{c}1 \\
( \pm 1,15)\end{array}$ & \multirow{2}{*}{0,550} \\
\hline & G2 & $\begin{array}{c}6,85 \\
( \pm 2,54)\end{array}$ & $\begin{array}{c}4,42 \\
( \pm 2,99)\end{array}$ & $\begin{array}{c}3,42 \\
( \pm 3,4)\end{array}$ & $\begin{array}{c}1,71 \\
( \pm 1,38)\end{array}$ & \\
\hline
\end{tabular}

Fonte: O autor

49\% das pacientes. Com o aprendizado errôneo, não há melhora do controle vesical e a paciente perde o estímulo para realizar um tratamento potencialmente útil no futuro.

Nesses casos, o tratamento com o BF é o mais indicado, pois ele permite visualizar a execução dos exercícios, isolar corretamente os MAP, maximizando os benefícios dos programas de TMAP, prevenindo agravamento do quadro e possibilitando melhor evolução no tratamento (AGOSTINHO; BERTOTTO, 2009; IBRAHIM et al., 2015).

Em todas as variáveis analisadas no presente estudo, os valores se mantiveram estáveis após a terceira e a quarta avaliações, ou seja, não houve regressão do tratamento nem evolução significativa do mesmo. É importante lembrar que no período entre a terceira e a quarta avaliação, a paciente foi orientada a continuar a realização dos EMAP em domicílio sem o acompanhamento profissional, por meio de um folder explicativo e orientações verbais, durante dois meses.

A Associação Portuguesa de Urologia (2013) destaca que as alterações comportamentais são necessárias para o sucesso terapêutico, devendo a paciente atentar-se à ingestão de líquidos, à exclusão de alimentos excitantes para a bexiga, à micção temporizada, além da prevenção de comorbidades associadas à IU, como alterações neurológicas, patologias urológicas e ginecológicas, afecções osteoarticulares (BRAVO, 2014), doença pulmonar, diabetes, demência e fibrilação atrial (DOGAN et al., 2015). No entanto, conforme o estudo de Sherburn et al. (2011), tais medidas apresentam poucos resultados positivos quando realizadas isoladamente, devendo ser associadas ao TMAP.

Resende e Stüpp (2015) também destacam a importância da execução dos exercícios com regularidade que, por meio de adaptações neurais e do potencial de hipertrofia muscular, transformam o AP 
em um rígido apoio estrutural, eficaz na prevenção e na melhora das perdas urinárias.

No estudo de Ibrahim et al. (2015), foram realizadas 24 sessões de TMAP por meio do BF em pacientes com IUE e com incontinência fecal e, quando comparadas ao grupo controle, o qual recebeu apenas orientações verbais sobre a execução dos EMAP em domicílio, observou-se melhora na força muscular em $72,2 \%$ das pacientes tratadas para apenas $31,8 \%$ no grupo controle, comprovando a melhor eficácia do treinamento clínico em relação ao domiciliar. Além disso, Bo e Herbert (2013) afirmam em seu estudo que mais de $30 \%$ das mulheres com disfunções no AP não são capazes de contraí-lo corretamente mesmo após treinamento individual.

No presente estudo, ambas as formas de intervenção descritas não apresentaram evolução significativa na quarta avaliação na maioria das variáveis. Este resultado pode ser justificado pelos achados descritos anteriormente, pelo fato de não ter sido possível analisar o real comprometimento por parte das participantes na realização dos EMAP e nas mudanças dos hábitos de vida, uma vez que o TMAP pode ter sido realizado de maneira insuficiente ou ineficaz para permitir maior evolução do tratamento.

Ainda assim, houve permanência do ganho de força muscular das fibras rápidas e maior tempo de contração das fibras lentas sobre ambos os grupos quando comparados os resultados com a primeira avaliação. Embora o G1 tenha apresentado resultados mais significativos em relação às fibras lentas, o mesmo não foi observado estatisticamente em relação às outras variáveis e nem durante a comparação inter-grupos. Portanto, é possível concluir que ambas as técnicas foram eficazes no processo de hipertrofia muscular, refletindo em diminuição das perdas miccionais mensais e na melhora da QV das participantes.

Apesar dos resultados estatisticamente significativos demonstrados, não foi possível observar diferença entre os grupos G1 e G2, bem como a evolução significativa do tratamento na terceira e quarta avaliações para as variáveis analisadas. Sugerese, portanto, estudos com amostras maiores para confirmação dos resultados.

\section{Conclusão}

Foi possível concluir que ambas as técnicas fisioterapêuticas propostas para o tratamento da IU em mulheres na menopausa foram eficazes na redução da frequência das perdas miccionais mensais e no ganho de força muscular das fibras rápidas, com consequente aumento na QV em tratamentos a curto, médio e longo prazo. Porém somente o BF apresentou eficácia sobre o tempo de contração mantida, sugerindo tratamento por meio deste método quando o objetivo for de melhorar essa variável.

\section{AGRADECIMENTOS}

À professora Ana Tereza Bittencourt, que foi de grande auxílio na análise dos resultados obtidos e, consequentemente, conclusão deste trabalho.

\section{Referências}

Agostinho, A. D.; BeRTOTTO, A. Biofeedback aplicado ao tratamento das incontinências urinárias. In: PALMA, P. C. R. Urofisioterapia: aplicações clínicas das técnicas fisioterapêuticas nas disfunções miccionais e do assoalho pélvico. São Paulo: P. Parma, 2009. Cap. 24, p. 255-267.

ALBA, D. V.; ALARMA, S. B.; REINA, G. R.; RODRÍGUEZ, J. C. Incontinência Urinaria. Medicine, Madrid, v. 10, n. 83, p. 5612-5618, 2011.

AMARAL, M. O. P.; COUTINHO, E. C.; NELAS, P. A. A. B.; CHAVES, C. M. B.; DUARTE, J. C. Risk factors associated with urinary incontinence in Portugal and the quality of life of affected women. International Journal of Gynecology and Obstetrics, Viseu, v. 131, p. 82-86, 2015.

ASSOCIAÇÃO PORTUGUESA DE UROLOGIA. Incontinência urinária. Disponível em: <http://www. apurologia.pt/incontinencia/incontinencia_2013/ Dossier_Imprensa_Incontinencia_Urinaria.pdf $>$. Acesso em: 15 out. 2015.

BERTOLDI, J. T.; GHISLERI, A. Q.; PICCININI, B. M. Fisioterapia na incontinência urinária de esforço: revisão de literatura. Revista Cinergis, Joinville, v. 15, n. 4, p. 1-6, 2014.

BO, K.; HERBERT, R. D. There is not yet strong evidence that exercise regimens other than pelvic floor muscle training can reduce stress urinary incontinence in women: a systematic review. Journal of Physiotherapy, Camberwell, v. 59, p. 159-168, 2013.

BRAVO, C. V. Protocolo diagnóstico y terapêutico de la incontinencia y del síndrome vesical irritativo. Medicine, Madrid, v. 11, n. 62, p. 3705-3709, 2014. 
BUMP, R. C.; MATTIASSON, A.; BØ, K.; BRUBAKER, L. P.; DELANCEY, J. O.; KLARSKOV, P.; SHULL, B. L.; SMITH, A. R. B. The standardization of terminology of female pelvic organ prolapse and pelvic floor dysfunction. American Journal of Obstetrics and Gynecology, Madison, v. 175, p. 1017, 1996.

CORREA, J. N.; MOREIRA, B. P.; GARCEZ, V F. Ganho de força muscular do diafragma pélvico após utilização dos métodos Pilates ou Kegel em pacientes com incontinência urinária de esforço. Revista Uningá, Maringá, v. 23, n.2, p. 11-17, 2015.

CURI, V. S. A influência do método Pilates nas atividades de vida diária de idosas. 2009. 68 f. Dissertação (Mestrado em Gerontologia Biomédica) - Pontifícia Universidade Católica do Rio Grande do Sul, Porto Alegre.

DINIZ, M. F.; VASCONCELOS, T. B.; PIRES, J. L. V. R.; NOGUEIRA, M. M.; ARCANJO, G. N. Avaliação da força muscular do assoalho pélvico em mulheres praticantes de Mat Pilates. Manual Therapy, Posturology \& Rehabilitation Journal, Fortaleza, v. 12, p. 406-420, 2014.

DOGAN, Z.; YALCIN, A.; ATMIS, V; CENGIZ, O.K.; ARAS, S.; VARLI, M.; CINAR, E.; ATLI, T. The prevalence of urinary incontinence and factors associated with urinary incontinence in community dwelling older Turkish people. European Geriatric Medicine, Turkey, v. 6, n. 5, p. 417-21, 2015.

FERREIRA, M.; SANTOS, P. Princípios da fisiologia do exercício no treino dos músculos do pavimento pélvico. Acta Urológica, Lisboa, v. 26, n. 3, p. 31-38, 2009.

FITZ, F. F.; COSTA, T. F.; YAMAMOTO, D. M.; RESENDE, A. P. M.; STÜPP, L.; SARTORI, M. G. F.; GIRÃO, M. J. B. C.; CASTRO, R. A. Impacto do treinamento dos músculos do assoalho pélvico na qualidade de vida em mulheres com incontinência urinária. Revista de Associação Médica Brasileira, São Paulo, v. 58, n. 2, p. 155-159, 2012a.

FITZ, F. F.; RESENDE, A. P. M.; STÜPP, L.; COSTA, T. F.; SARTORI, M. G. F.; GIRÃO, M. J. B. C.; CASTRO, R. A. Efeito da adição do biofeedback ao treinamento dos músculos do assoalho pélvico para tratamento da incontinência urinária de esforço. Revista Brasileira de Ginecologia e Obstetrícia, São Paulo, v. 34, n. 11, p. 505-10, 2012 b.
GIRALDO, P. C.; RETT, M. T.; GABIATTI, J. R. E.; JUNIOR, J. E.; GONÇALVES, A. K. S.; AMARAL, R. L. Repercussões da eletroestimulação intravaginal no ecossistema vaginal. In: PALMA, P. C. R. Urofisioterapia: aplicações clínicas das técnicas fisioterapêuticas nas disfunções miccionais e do assoalho pélvico. São Paulo: P. Parma, 2009. Cap. 34, p. 365-372.

GUIRRO, E. C. O. Eletroterapia no tratamento da incontinência urinária de esforço. In: PALMA, P. C. R. Urofisioterapia: aplicações clínicas das técnicas fisioterapêuticas nas disfunções miccionais e do assoalho pélvico. São Paulo: P. Parma, 2009. Cap. 23, p. 247-254.

HIRAKAWA, T.; SUZUKI, S.; KATO, K.; GOTOH, M.; YOSHIKAWA, Y. Randomized controlled trial of pelvic floor muscle training with or without

biofeedback for urinary incontinence. International Urogynecological Journal, London, v. 24, p. 13471354, 2013.

HAYLEN, B. T.; de RIDDER, D.; FREEMAN, R. M.; SWIFT, S.E.; BERGHMANS, B.; LEE, J.; MONGA, A.; PETRI, E.; RIZK, D. E.; SAND, P.K.; SCHAER, G. N. An International Urogynecological Association (IUGA)/International Continence Society (ICS) joint report on the terminology for female pelvic floor dysfunction. Journal of the Association of Chartered Physiotherapists in Women's Health, London, v. 110, p. 33-57, 2012.

HERDERSCHEE, R.; HAY-SMITH, E. J. C.; HERBISON, G. P.; ROOVERS, J. P.; HEINEMAN, M. J. Feedback or Biofeedback to augment pelvic floor muscle training for urinary incontinence in women (Review). Cochrane Database of Systematic Reviews, Amsterdam, v. 7, p. 1-150, 2011.

IBRAHIM, I. K.; HAMEED, M. M. A.; TAHER, E. M.; SHAHEEN, E. M.; ELSAWY, M. S. A. G. Efficacy of biofeedback-assisted pelvic floor muscle training in females with pelvic floor dysfunction. Alexandria Journal of Medicine, Alexandria, v. 51, p. 137-142, 2015.

JUSTINA, L. B. D. Prevalência de incontinência urinária feminina no Brasil: uma revisão sistemática. Revista Inspirar: Movimento \& Saúde, Tubarão, v. 6, n. 3, p. 7-13, 2013. 
MARÉS, G.; OLIVEIRA, K. B.; PIAZZA, M. C.; PREIS, C.; NETO, L. B. A importância da estabilização central no método Pilates: uma revisão sistemática. Revista Fisioterapia em Movimento, Curitiba, v. 25, n. 2, p. 445-451, 2012.

MORENO-VECINO, B.; BLÁZQUEZ, A. A.; PEDRERO-CHAMIZO, R.; ALCÁZAR, J.; GÓMEZCABELlO, A.; PÉREZ-LÓPEZ, F.; GONZÁlEZGROSS, M.; CASAJÚS, J. A.; ARA, I. Associations between obesity, physical fitness, and urinary incontinence in non-institutionalized postmenopausal women: The elderly EXERNET multi-center study. Maturitas, Madrid, v. 82, n. 2, p. 208-214, 2015.

QASEEM, A.; DALlAS, P.; FORCIEA, M. A.; STARKEY, M.; DENBERG, T. D.; SHEKELLE, P. Nonsurgical management of urinary incontinence in women: a clinical practice guideline from theAmerican College of Physicians. Annals of Internal Medicine, Philadelphia, v. 161, n. 6, p. 429-440, 2014.

RESENDE, A. P. M.; STÜPP, L. Fisioterapia. In: GIRÃO, M. J. B. C; SARTORI, M. G. F.; RIBEIRO, R. M.; CASTRO, R. A.; BELLA, Z. I. K. J. Tratado de uroginecologia e disfunções do assoalho pélvico. São Paulo: Manole, 2015. Cap. 12, p. 181-190.

SANTOS, F. D. R. P. Análise entre a técnica de cinesioterapia isolada e associada ao biofeedback no tratamento da incontinência urinária de esforço: estudo de dois casos. Revista Movimenta. Maranhão, v. 8, n. 1, p. 80-86, 2015.

SHERBURN, M.; BIRD, M.; CAREY, M.; BO, K.; GALEA, M. P. Incontinence improves in older women after intensive pelvic floor muscle training: an assessorblinded randomized controlled trial. Neurology and Urodynamics, Melbourne, v. 30, n. 3, p. 317-24, 2011.

SILVA, A. C. L. G.; MANNRICH, G. Pilates na reabilitação: uma revisão sistemática. Revista Fisioterapia em Movimento, Curitiba, v. 22, n. 3, p. 449-455, 2009.

SOCIEDADE BRASILEIRA DE HIPERTENSÃO. Diretrizes brasileiras de hipertensão IV. Revista Hipertensão, São Paulo, v. 13, n. 1, p. 1-68, 2010.

TAMANINI, J. T. N.; DAMBROS, M.; D’ANCONA, C. A. L.; PALMA, P. C. R.; JÚNIOR, N. R. N. Validação para o português do International Consultation on Incontinence Questionnaire: short form (ICIQ-SF). Revista de Saúde Pública, São Paulo, v. 38, n. 3, 2004.
TOURNIER, M. B. Comportamento da musculatura perineal frente ao método Pilates em bola suiça: um estudo com acadêmicas da UNESC de Criciúma/SC. 2011. 68 f. Monografia (Graduação em Fisioterapia) Universidade do Extremo Sul Catarinense, Criciúma. 
Eficácia do método Pilates e do Biofeedback manométrico em mulheres na menopausa com incontinência...

\section{APÊNDICE 01 - TERMO DE CONSENTIMENTO LIVRE E ESCLARECIDO - TCLE}

Título do Projeto: Avaliação da qualidade de vida de pacientes com incontinência urinária submetidas a diferentes técnicas de tratamento fisioterapêutico

Pesquisador responsável: Juliana Cristina Frare (045XXXXXXXX)

Colaboradores com telefones de contato: Beatriz Gavassa de Araújo (045XXXXXXXX); Caroline Danielli (045XXXXXXXX); Elisa Pinheiro Schrader (045XXXXXXXX)

A incontinência urinária (IU) é definida como a queixa de qualquer perda involuntária de urina. Atualmente, é considerada um problema de saúde comum entre as mulheres e determina repercussões negativas nas questões sociais, médicas, psicológicas e econômicas, incluindo isolamento social e baixa autoestima, afetando negativamente a qualidade de vida.

Convidamos você a participar de nossa pesquisa que tem o objetivo de avaliar a qualidade de vida de pacientes com incontinência urinária submetidas à fisioterapia com Método Pilates ou uma técnica terapêutica, mais conhecida como biofeedback, que utiliza instrumentos eletrônicos para medir com precisão, processar e retroalimentar as informações tanto para o indivíduo quanto para seu terapeuta. Para isso, inicialmente você responderá uma ficha de Avaliação Fisioterapêutica em Incontinência Urinária e o Questionário de Incontinência Urinária ICIQ_SF. Na sequência, em um ambiente privativo, será realizada uma avaliação clínica individual na região genital feminina através de um exame físico e verificação da pressão muscular do assoalho pélvico (MAP). Os grupos de tratamento serão divididos de forma aleatória através de um sorteio.

Se você fizer parte do grupo que irá ser submetido à técnica do Método Pilates, suas sessões serão realizadas em grupo. Cada sessão terá duração de 50 minutos e você irá realizar diferentes exercícios em solo.

Se você fizer parte do grupo que irá ser submetido à técnica de biofeedback, sua sessão será realizada individualmente e terá um tempo de duração de 25 minutos.

Ao final das 22 sessões, você será reavaliada para que possamos verificar a eficiência das técnicas terapêuticas aplicadas.

O participante desta pesquisa corre risco baixo, tendo em vista que os exercícios serão realizados no limite de cada paciente, não predispondo qualquer tipo de lesão. Considerando-se o risco de constrangimento para as participantes devido aos procedimentos que serão realizados para as avaliações, os mesmos serão previamente explicados em detalhes e, posteriormente, com o consentimento das pacientes, realizados individualmente em ambiente adequado. Caso a paciente refira dor ou desconforto após cada etapa (avaliações e sessões de aplicação dos métodos), ele será encaminhado para o devido tratamento fisioterapêutico na Clínica de Fisioterapia da Unioeste. Para algum questionamento, dúvida ou relato de algum acontecimento os pesquisadores poderão ser contatados a qualquer momento.

Sua participação é essencial para a pesquisa que tem como objetivo verificar qual desses dois métodos terapêuticos é mais eficaz para tratar a população feminina que sofre de incontinência urinária, ajudando assim, a melhorar a qualidade de vida destas mulheres.

Este termo de consentimento livre e esclarecido (TCLE) será entregue em duas vias, sendo que uma ficará com você e outra com os pesquisadores. Você participante, não pagará nem receberá nada para participar do estudo. Será mantida a confidencialidade do participante e os seus dados serão utilizados somente para fins científicos. Você poderá cancelar sua participação a qualquer momento.

Caso necessite de maiores informações pode entrar em contato com o comitê de ética no telefone 3220-3272. Caso ocorra algum imprevisto durante a avaliação da pesquisa você será amparado da melhor forma possível, até mesmo se for necessário que seja chamado o SAMU. 
Declaro estar ciente do exposto e desejo participar da pesquisa.

Nome do sujeito de pesquisa ou responsável:

Assinatura:

Eu, Juliana Cristina Frare, declaro que forneci todas as informações do projeto ao participante e/ ou responsável.

Cascavel, de de 20 


\section{APÊNDICE 02 - FICHA DE COLETA DE DADOS}

\begin{tabular}{|c|c|c|}
\hline 8 \\
8 & $\begin{array}{l}\text { AVALIÇÃO DA QUALIDADE DE VIDA DE PACIENTES COM } \\
\text { INCONTINÊNCIA URINÁRIA SUBMETIDAS Ȧ DIFERENTES } \\
\text { TÉCNICAS DE TRATAMENTO FISIOTERAPÊUTICO }\end{array}$ & 8 \\
\hline
\end{tabular}

\section{FICHA DE COLETA DE DADOS INICIAL}

Data:

Dados Pessoais:

Nome:

Data de nascimento:

Endereço:

Telefone:

Profissão:

Peso:

Altura:

( ) Tabagista

( ) Já realizou algum tratamento para a incontinência anteriormente

( ) Qual tratamento?

( ) Há quanto tempo realizou este tratamento?

Cor: ( ) Branca ( ) Negra ( ) Amarela

Hábitos Urinários Frequência miccional: dia: noite:

（ ) gotas pós-micção （ ) sensação de queimação ou ardência
( ) disúria
( ) força abdominal
( ) atraso na micção

( ) dificuldade de segurar urina - urgência

Exame da Incontinência

Quando começou?

Frequência da perda?

Incontinência: ( ) diurna ( ) noturna ( ) esforço ( ) contínua

Proteção: ( ) permanente ( ) ocasionalmente

Tipo de proteção: ( ) protetor de calcinha ( ) absorvente ( ) fraldas( ) outros

( ) troca peças íntimas $\quad \mathrm{N}^{\circ}$ trocas / dia?

Quantidade perdida: ( ) Jato ( ) Gota a gota ( ) Completa ( ) Insensível 
( ) Outras:

Situações em que ocorre perda - IUE

( ) Tosse ( ) Espirro ( ) Riso ( ) Subir escadas ( ) Evacuar ( ) Andar ( ) Carregar pesos

( ) Dançar ( ) Relação Sexual ( ) Trocar de posição ( ) Correr ( ) Outros

Situações em que ocorre perda - IUM

( ) Estresse ( ) Frio ( ) Barulho de Torneira ( ) Mãos na água

( ) Urgência para ir ao banheiro ( ) Outros

Exame físico:

Perineômetro:

\begin{tabular}{|c|c|c|}
\hline & Contrações rápidas & Contrações mantidas \\
\hline $1^{\mathrm{a}}$ leitura & & \\
\hline $2^{\mathrm{a}}$ leitura & & \\
\hline $3^{\mathrm{a}}$ leitura & & \\
\hline
\end{tabular}

Avaliações objetivas da paciente:

Quanto sua qualidade de vida em relação a Incontinência Urinária

$0 \_1 \_2 \_3 \quad 4 \quad 5 \quad 6 \quad 6 \quad 7 \quad 8 \quad 9 \quad 9 \quad 10$

Excelente

Péssima

Quanto à sensação de umidade:

$\begin{array}{lllllll}1 & 2 & 3 & 4 & 5\end{array}$

Seco

Molhado

Quanto à sensação e incômodo na vida diária e atividade social:

$\begin{array}{llllll}1 & 2 & 3 & 4 & 5\end{array}$

Ausência de incômodo

Muito incômodo / alteração vida social 


\section{APÊNDICE 03 - FOLDER INFORMATIVO PARA EMAP}

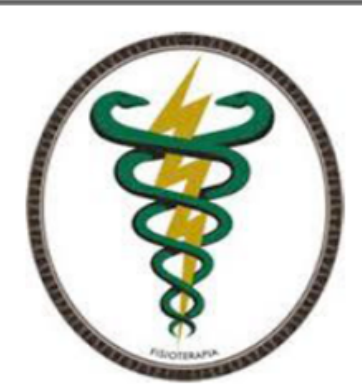

\section{INCONTINÊNCIA} URINÁRIA

\section{VOCÊ SABE O QUE É?}

CENTRO DE REABILITAÇÃO FÍSICA - CRF

CLÍNICA-ESCOLA

DE FISIOTERAPIA

(45) 3220-3236

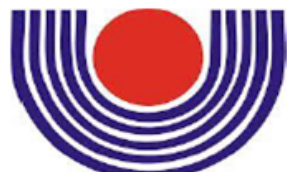

unioeste

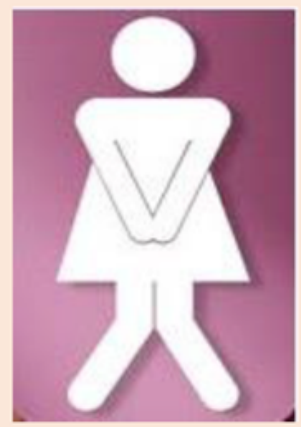

Incontinência urinária é toda perda involuntária de urina, a qual pode causar problema social, higiênico e psicológico.

Para evitá-la é fácil, basta seguir algumas orientações:

- Eduque sua bexiga

Estabeleça periodos para ir ao banheiro, como a cada 2 horas. Não espere a vontade aumentar.

\section{- Tenha cuidado com líquidos}

Sucos citricos, refrigerantes e alimentos que contêm cafeína estimulam a vontade de ir ao banheiro.

\section{- Mantenha boa hidratacão}

Principalmente durante o dia. Evite beber muito liquido durante a noite. Quanto maior a ingesta liquida, maior a frequência de idas ao banheiro.

- Controle o peso e faça sempre uma atividade física A obesidade também aumenta a chance de incontinenncia urinária.

- Evite fumar ou ingerir bebidas alcoólicas Ambos também estimulam a ação da bexiga.

- Treine sempre os músculos do assoalho pélvico. Ou seja, os músculos responsáveis pela sustentação dos órgãos internos e que controlam a saida da urina.

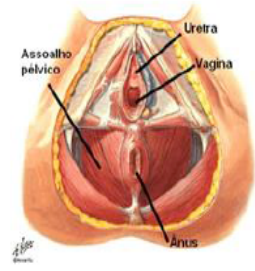

Siga no mínimo 2 dos exercícios programados pelo menos 3 vezes por semana:
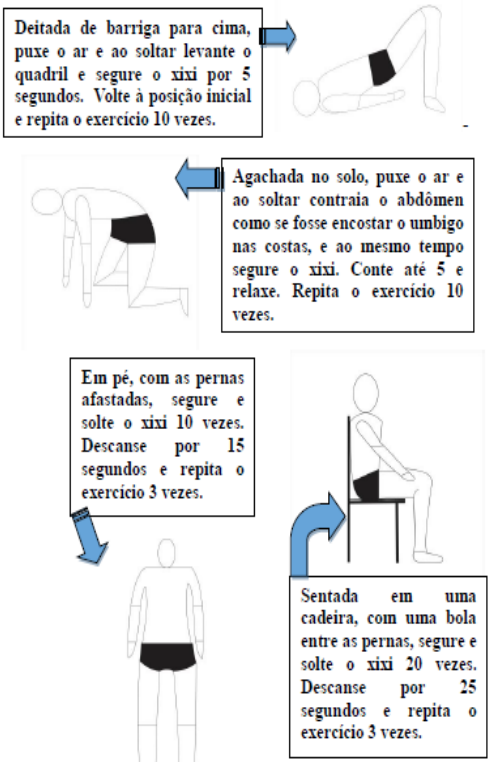


\section{ANEXO 01 - QUESTIONÁRIO INCONTINÊNCIA URINÁRIA}

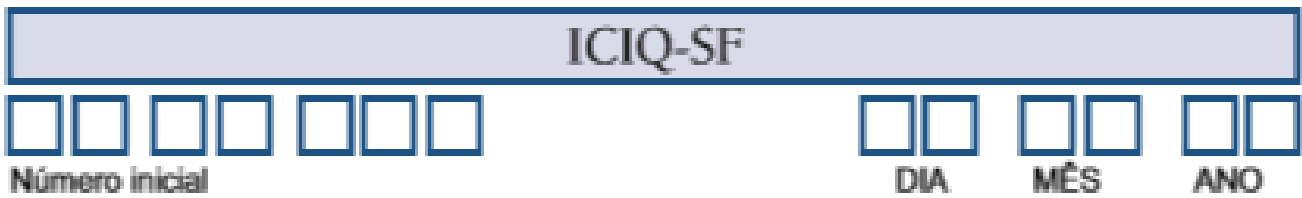

Muitas pessoas tėm por vezes perdas de urina. Estamos a tentar perceber quantas pessoas tềm perdas de urina e se esta situação as incomoda. Agradeciamos que respondesse às perguntas que se seguem, tendo em conta aquilo que tem sentido, em média, nas ÚLTIMAS QUATROSEMANAS.

1. Preencha com a sua data de nascimento, s.f.f.:

2. Sexo (assinale uma):

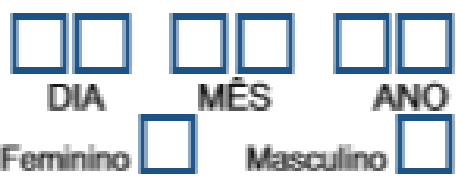

3. Com que frequência tem perdas de urina? (Escolha uma opçio)

\begin{tabular}{|c|c|}
\hline \multirow{2}{*}{\multicolumn{2}{|c|}{ nunce }} \\
\hline & \\
\hline uma vez por semana ou menos & 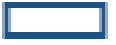 \\
\hline duas ou três vezes por semana & \\
\hline uma vez por dia & \\
\hline várias vezes por dia & \\
\hline constantemente & - \\
\hline
\end{tabular}

4. Gostariamos de saber a quantidade de urina que acha que perde. Que quantidade de urina costuma perder (quer use ou nä́o proteç̧äo) (escollha uma opçäo)

$\begin{array}{rlr}\text { nenhuma } & \square & 0 \\ \text { uma quantidade pequena } & & 2 \\ \text { uma quantidade moderada } & & 4 \\ \text { uma grande quantidade } & & \end{array}$

5. No geral, a perda de urina interfere muito no seu dia-a-dia? Faça um circulo entre 0 (nada) e 10 (bastante)

$\begin{array}{llllllllll}0 & 1 & 2 & 3 & 4 & 5 & 6 & 7 & 8 & 9 \\ \text { nada } & & & & & & & & & \\ \text { bastante }\end{array}$

pontuação ICIQ: somar pontuaçóes 3+4+5

6. Quando tem perdas de urina? (Escolha todas as oppōes que se aplicam a si) nunca - nāo tem perdas de urina tem perdas de urina antes de conseguir chegar à casa de banho tem perdas de urina quando tosse ou espirra tem perdas de urina quando está a dormir tem perdas de urina quando está fisicamente activo(a) / a fazer exercicio tem perdas de urina quando acabou de urinar e está vestido(a) tem perdas de urina sem razão aparente tem perdas de urina constantemente 